\title{
Differences in muscle strength after ACL reconstruction do not influence cardiorespiratory responses to isometabolic exercise
}

\author{
Marília S. Andrade ${ }^{1}$, Claudio A. B. Lira ${ }^{2}$, Rodrigo L. Vancini ${ }^{3}$, \\ Fernanda P. Nakamoto ${ }^{1}$, Moisés Cohen ${ }^{4}$, Antonio C. Silva ${ }^{1}$
}

\begin{abstract}
Objectives: To investigate whether the muscle strength decrease that follows anterior cruciate ligament (ACL) reconstruction would lead to different cardiorespiratory adjustments during dynamic exercise. Method: Eighteen active male subjects were submitted to isokinetic evaluation of knee flexor and extensor muscles four months after ACL surgery. Thigh circumference was also measured and an incremental unilateral cardiopulmonary exercise test was performed separately for both involved and uninvolved lower limbs in order to compare heart rate, oxygen consumption, minute ventilation, and ventilatory pattern (breath rate, tidal volume, inspiratory time, expiratory time, tidal volume/inspiratory time) at three different workloads (moderate, anaerobic threshold, and maximal). Results: There was a significant difference between isokinetic extensor peak torque measured in the involved (116.5 $\pm 29.1 \mathrm{Nm})$ and uninvolved $(220.8 \pm 40.4 \mathrm{Nm})$ limbs, $\mathrm{p}=0.000$. Isokinetic flexor peak torque was also lower in the involved limb than in the uninvolved limb $(107.8 \pm 15.4$ and $132.5 \pm 26.3 \mathrm{Nm}, \mathrm{p}=0.004$, respectively). Lower values were also found in involved thigh circumference as compared with uninvolved limb $(46.9 \pm 4.3$ and $48.5 \pm 3.9 \mathrm{~cm}, \mathrm{p}=0.005$, respectively). No differences were found between the lower limbs in any of the variables of the incremental cardiopulmonary tests at all exercise intensities. Conclusions: Our findings indicate that, four months after ACL surgery, there is a significant deficit in isokinetic strength in the involved limb, but these differences in muscle strength requirement do not produce differences in the cardiorespiratory adjustments to exercise. Based on the hypotheses from the literature which explain the differences in the physiological responses to exercise for different muscle masses, we can deduce that, after 4 months of a rehabilitation program after an ACL reconstruction, individuals probably do not present differences in muscle oxidative and peripheral perfusion capacities that could elicit higher levels of peripheral cardiorepiratory stimulus during exercise.
\end{abstract} Keywords: rehabilitation; anterior cruciate ligament; isokinetic testing; physical therapy; oxygen uptake; ventilatory response.

\section{HOW TO CITE THIS ARTICLE}

Andrade MS, Lira CAB, Vancini RL, Nakamoto FP, Cohen M, Silva AC. Differences in muscle strength after ACL reconstruction do not influence cardiorespiratory responses to isometabolic exercise. Braz J Phys Ther. 2014 Mar-Apr; 18(2): 144-151. http://dx.doi. org/10.1590/S1413-35552012005000153

\section{Introduction}

The maintenance of homeostasis during exercise requires different physiological adjustments. The final response to moderate exercise is characterized by a steady state of physiological variables ${ }^{1}$. The physiological variables in the steady state of exercise tend to match the metabolic rate while the respiratory exchange ratio and the partial arterial pressures of carbon dioxide and oxygen remain close to resting levels ${ }^{2}$. Blood lactate concentration also remains near resting values during the steady state of moderate exercise $^{2}$. Consequently, minute ventilation $\left(\dot{\mathrm{V}}_{\mathrm{E}}\right)$ is proportional to oxygen uptake $\left(\mathrm{V}_{2}\right)$ and carbon dioxide production $\left(\dot{\mathrm{VCO}}_{2}\right)^{1}$. In the same way as
$\dot{\mathrm{V}}_{\mathrm{E}}$, cardiovascular responses also adapt to exercise intensity under the command of the autonomic nervous system ${ }^{1}$.

Under certain conditions, however, the proportionality between ventilatory and cardiovascular responses to exercise intensity is lost. For instance, exercise performed by different muscle masses at the same level of $\dot{\mathrm{V}} \mathrm{O}_{2}$ leads to significantly higher $\dot{\mathrm{V}}_{\mathrm{E}}$ and heart rate (HR) for a smaller muscle mass ${ }^{2,3}$. In the attempt to explain such findings, different hypotheses were formulated to elucidate the cardiorespiratory control during exercise, namely central command, peripheral chemoreceptors, and spinal reflexes ${ }^{4}$.

\footnotetext{
${ }^{1}$ Department of Physiology, Universidade Federal de São Paulo (UNIFESP), São Paulo, SP, Brazil

${ }^{2}$ Department of Human and Exercise Physiology, Faculty of Physical Education, Universidade Federal de Goiás (UFG), Goiânia, GO, Brazil

${ }^{3}$ Center for Physical Education and Sport, Universidade Federal do Espírito Santo (UFES), Vitória, ES, Brazil

${ }^{4}$ Department of Orthopedics and Traumatology, UNIFESP, São Paulo, SP, Brazil

Received: 04/04/2013 Revised: 08/25/2013 Accepted: 10/23/2013
} 
According to the central command hypothesis, the changes in cardiorespiratory variables that occur during exercise may be partly explained by the 'radiation' of activity in central neurons innervating exercising muscles to the respiratory and cardiovascular control areas ${ }^{3}$, serving as a feedforward control mechanism that provides an adequate ventilatory response to the metabolic demands of exercise ${ }^{5}$. Cardiorespiratory and locomotor responses are proportional, and only a fine adjustment feedback system (via humoral or peripheral neurogenic mechanisms) is necessary to assure an adequate ventilatory response to the metabolic demands of exercise. Several authors have suggested that central command is responsible for the ventilatory control in different types of exercise with different muscle mass, such as in subjects with unilateral atrophy $^{3}$, pharmacologically induced paralysis ${ }^{6}$ or during imagination of exercise without performing it $^{7}$. In all these models there is a strong correlation between ventilatory response and the level of central command.

The chemoreceptor hypothesis (aortic and carotid bodies) has been supported, especially for hyperpnea related to exercise when there is metabolic acidosis ${ }^{8}$. Several different stimuli may be responsible for increasing the contribution of the peripheral chemoceptors to ventilation during exercise, as long as a degree of hypoxia is present ${ }^{9}$. In relation to the spinal reflex theory, peripheral neurogenic stimuli originated in receptors located inside or outside the exercising muscles are related to the perception of muscle movement, position of the joints, muscular metabolic changes and changes in vascular conductance of exercising muscles ${ }^{1,4,9}$. Different authors have proposed that exaggerated cardiorespiratory response in relation to the metabolic demand observed during exercise with different muscle masses is determined by intramuscular metabolic receptors sensitive to local metabolic changes in the same absolute workload, exercise performed with a smaller muscle mass produces more intense metabolic responses ${ }^{2,10}$. Others suggest that cardiorespiratory control is not correlated with the local metabolic changes, but with variation in peripheral blood flow, obstruction of venous flow for exercising muscles increases the ventilatory response and when the arterial flow is also blocked the ventilatory response diminishes, indicating that local vascular conductance plays an important role in the ventilatory adjustments to exercise ${ }^{11}$.

Regardless of the mechanisms that could explain higher cardiorespiratory responses to exercise performed by smaller muscle masses, the knowledge of these differences may be useful in orthopedic rehabilitation procedures. In clinical practice, a common situation that results in muscular (strength, power, and endurance) and sensory deficits is anterior cruciate ligament (ACL) reconstruction ${ }^{12}$. After surgery, the patient presents a significant loss in muscle strength, especially in the quadriceps muscle, even 6 months after ACL reconstruction ${ }^{13,14}$. To identify successful outcomes after surgery, members of several international sports medicine associations (American Orthopedic Society for Sports Medicine, the European Society for Sports Traumatology, Surgery, and Knee Arthroscopy, and the American Physical Therapy Association) agree with the following criteria: thigh muscle strength recovery (strength level greater than $90 \%$ of the uninjured limb), absence of giving way, return to sports, no more than mild knee joint effusion, and using patientreported outcomes. Therefore, given that patients who were submitted to ACL reconstruction presented significantly smaller thigh mass, it is possible that they presented higher cardiorespiratory responses to isometabolic unilateral exercise during the process of rehabilitation to recover lower limb muscle mass. If so, these differences may be used as criteria to infer the extent of muscular deficiency and to guide orthopedic and sports rehabilitation procedures. Previous studies have demonstrated differences in cardiorespiratory responses to isometabolic exercise performed by different muscle masses ${ }^{15,16}$, however these studies are from the 1990s and there are no studies comparing lower limb responses after unilateral ligament reconstruction.

Thus, the aim of the study was to verify whether there are differences in cardiorespiratory adjustments to dynamic exercise performed by both uninvolved and involved lower limbs after unilateral ACL reconstruction. We hypothesized that, due to differences in muscle mass, cardiorespiratory adjustments to dynamic exercise are exacerbated when both involved and uninvolved limbs results are compared.

\section{Method}

\section{Subjects}

Eighteen physically active male subjects $(2.7 \pm 0.7$ hours/week of physical activity) participated in the study. Their mean age was $33 \pm 12$ years, height was $177 \pm 5 \mathrm{~cm}$, and body mass was $79 \pm 9 \mathrm{~kg}$. 
Subjects were submitted to intra-articular ACL reconstruction with patellar tendon graft performed by the same surgical team. Following the surgery, they participated in a standardized ACL rehabilitation program for both limbs, as previously described by Carey et al. ${ }^{17}$. Sessions were held three times a week. In addition to cruciate ligament tear, the lateral meniscus was involved in four subjects, the medial meniscus in seven subjects, and both lateral and medial menisci in one subject.

The subjects were informed about the purpose of the research and were asked to give their written consent to participate in the protocol approved by the Ethics Committee of Universidade Federal de São Paulo (UNIFESP), São Paulo, SP, Brazil (protocol 281/99). All experimental procedures were in accordance with the recommendations of the Declaration of Helsinki.

\section{Experimental procedures}

The entire evaluation lasted two consecutive days, starting four months after the surgery. This time period was chosen in order to minimize the possibility of pain or fear, which could influence the physiological responses to exercise and be harmful to the integrity of the graft. On the first day, the volunteers were submitted to isokinetic muscular evaluation of the knee flexor and extensor muscles. Measurement of the thigh circumference was taken and the knee range of motion (ROM) was assessed in order to verify the losses in muscular function caused by surgery. Thigh circumference and ROM were performed in a random order before the isokinetic test. Evaluation of the cardiorespiratory responses to exercise for both limbs (involved and uninvolved) was performed on the second day.

Isokinetic muscle test. Before isokinetic testing, a 5-min warm-up was performed on a cycle ergometer (Cybex Inc., Ronkonkoma, NY, USA) at a resistance level of $25 \mathrm{~W}$ followed by low-intensity dynamic stretching exercises for the hamstrings and quadriceps stretching exercises ${ }^{18}$. Following the warm-up, subjects were placed on the isokinetic dynamometer (Cybex - division of Lumex, Cybex 6000) to assess the concentric strength measurements for both involved and uninvolved lower limbs. Subjects assumed a seated position on the isokinetic dynamometer with their hips flexed at approximately $85 \mathrm{deg}$, measured by the scale in the isokinetic dynamometer chair. Standard stabilization straps were placed across the trunk, the waist, and the distal femur of the tested limb to minimize additional movement and to provide the same conditions for all participants.
The axis of the dynamometer was visually aligned with the lateral femoral condyle while the knees were flexed at $90 \mathrm{deg}$. The length of the lever arm was individually determined by the length of each individual's lower leg, and the resistance pad was placed proximal to the medial malleolus. Following the direct measurement at $30 \mathrm{deg}$ of knee extension, gravity correction procedures were applied according to the manufacturer's specifications to reduce the risk of inaccurate data. The knee flexion/extension range of motion was standardized from 5 to $95 \mathrm{deg}$, with full knee extension referenced as being 0 deg, following previous procedures ${ }^{18}$. Given that maximal torque output is not altered by small variations in range of motion during isokinetic activity testing ${ }^{18}$, this range of motion was chosen to prevent graft injury. The sequence of sides for each subject was randomized and all participants were tested on extension first.

As part of the familiarization process, the participants were given standard verbal instructions regarding the procedures and were allowed a few (no more than 5) sub-maximal practice attempts for each test condition. After 1 minute, the subjects were tested with a maximum of 5 repetitions performed at $60 \%$, with no rest period between knee extension and flexion assessments, and the results were recorded for analysis. This number of repetitions has been shown to have high reliability for isokinetic testing ${ }^{19}$. Consistent verbal commands were given by the examiner during each test to ensure maximal effort, and visual feedback of the recorded torque was provided during the test. All subjects were tested by the same examiner who was trained and experienced in the use of isokinetic testing devices. The evaluated parameters were peak torque (in $\mathrm{Nm}$ ), total work (in Joules), and set total work (in Joules) of the hamstrings and quadriceps. Contralateral deficits (in \%) [1 - (involved limb value/uninvolved limb value) *100] were calculated for all three variables measured and for both flexor and extensor muscles.

\section{Thigh circumference}

Thigh circumference was taken with a tape measure $10 \mathrm{~cm}$ above the superior pole of the patella, with the subject in a standing position and both feet on the floor.

\section{Knee range of motion}

Knee flexion and extension ROM in degrees were measured bilaterally in prone. For this purpose, the lateral femoral condyle was used as a landmark for the measurement of knee flexion and extension. The central pivot of a universal goniometer (CARCI, São 
Paulo, SP, Brazil) was placed over the midpoint of the lateral joint margin, with the stationary arm aligned with the great trochanter. The moving arm was then aligned with the lateral malleolus with the neutral position taken as zero. To measure knee flexion, the hip was initially at $0 \mathrm{deg}$ of extension, abduction, and adduction. In order to avoid knee flexion, the examiner held the lower limb and stabilized the femur to prevent hip rotation, abduction or adduction. To measure knee extension, the lower limb was extended. The previous precautions were taken to prevent compensations (i.e. adduction, abduction, and rotation). Measurements were carried out by a single experienced examiner.

\section{Cardiopulmonary incremental exercise test}

The comparative study between the limbs was performed using an isokinetic dynamometer Cybex 6000 in the isotonic mode. For this purpose, a special test was designed to measure the cardiorespiratory responses to exercise in closed kinetic chain, similar to the rehabilitation exercises. An adaptation in the equipment was done to allow extremity fixation to the lever system in closed kinetic chain (Figure 1). The subjects were kept in supine and performed active triple extension (ankle, knee, and hip), while flexion was passive. The protocol consisted of an initial workload of $15 \%$ of the involved limb, maximal isokinetic knee extensor peak torque (open kinetic chain) $($ mean $=21.6 \mathrm{Nm}$ and $\mathrm{SD}=7.4 \mathrm{Nm}$ ) for both limbs, with $10 \%$ increments every minute until volitional exhaustion, with a cadence of 45 cycles per minute guided by a metronome. Total test time was approximately 8 to 10 minutes for each limb. The test protocol was the same for both limbs to ensure that involved and uninvolved limbs were tested with

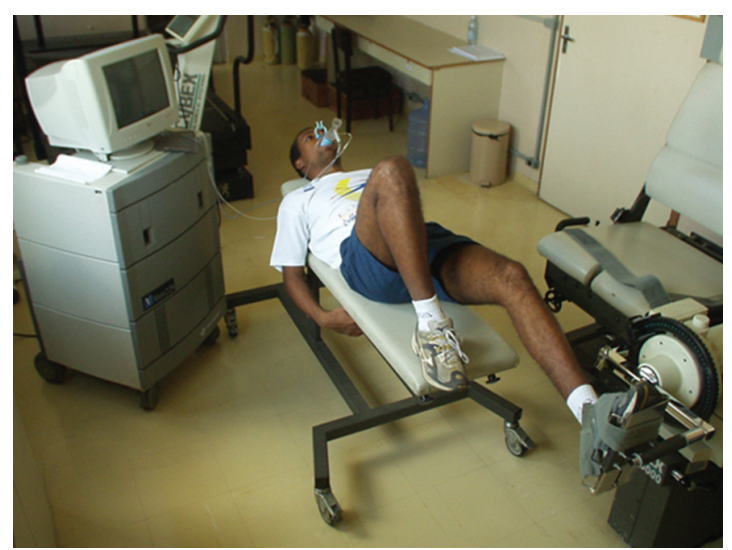

Figure 1. Cardiopulmonary incremental exercise test performed on isokinetic device. identical workloads. All subjects received a 60-min rest period between tests to prevent the build-up of fatigue. The sequence of lower limbs for each subject was randomized. During the test, subjects breathed through a two-way valve and the exhaled air was analyzed by a breath-by-breath metabolic system (Vmax 229, Sensor Medics, USA). The metabolic system was calibrated before each test according to the manufacturer's recommendations. HR was measured with a heart rate monitor (Vantage, Polar, Finland). For the purposes of the present study, the outcomes of interest were $\dot{\mathrm{V}} \mathrm{O}_{2}, \dot{\mathrm{V}}_{\mathrm{E}}$, ventilatory equivalent for $\mathrm{O}_{2}\left(\dot{\mathrm{V}}_{\mathrm{E}} / \dot{\mathrm{VO}}_{2}\right)$, ventilatory equivalent for $\mathrm{CO}_{2}\left(\dot{\mathrm{V}}_{\mathrm{E}} / \dot{\mathrm{V}} \mathrm{CO}_{2}\right)$, end-tidal pressure of $\mathrm{O}_{2}\left(\mathrm{PETO}_{2}\right)$, end-tidal pressure of $\mathrm{CO}_{2}\left(\mathrm{PETCO}_{2}\right)$, inspiration time $(\mathrm{Ti})$, expiration time $(\mathrm{Te})$, tidal volume $\left(\mathrm{V}_{\mathrm{T}}\right)$, respiratory drive $\left(\mathrm{V}_{\mathrm{T}} / \mathrm{Ti}\right)$, breathing rate $(f)$, and $\mathrm{HR}$. The anaerobic ventilatory threshold was indirectly estimated from breath-by-breath responses as the $\dot{\mathrm{VO}}_{2}$ at which $\mathrm{PETO}_{2}$ and the $\dot{\mathrm{V}}_{\mathrm{E}} / \dot{\mathrm{V}}_{2}$ systematically began to increase without simultaneous increase in the $\dot{\mathrm{V}}_{\mathrm{E}} / \mathrm{VCO}_{2}$ and decrease in $\mathrm{PETCO}_{2}^{8}$.

\section{Statistical analysis}

Sample size calculation was performed considering the type one error $(\alpha=0.05)$ and the type two error $(\beta=0.20)$. The calculations indicated that a sample of 16 subjects would be necessary to identify significant differences in minute ventilation. The results are expressed as mean (SD). As variables presented normal distributions according to the Shapiro-Wilk test, we used the paired Student t-test to compare the values for both limbs. The level of significance was set at $\mathrm{p}<0.05$. All statistical analyses were performed with Statistica, version 7.0 (Statsoft Inc, Tulsa, Oklahoma, USA).

\section{Results}

Four months after ACL surgery the involved limb was impaired when compared with the uninvolved limb for all measured muscle variables: thigh circumference $(-3.3 \%)$, range of motion $(-2.4 \%)$, isokinetic extensor peak torque $(-47.2 \%)$, isokinetic extensor total work $(-43.0 \%)$, isokinetic flexor peak torque $(-12.7 \%)$, and isokinetic flexor total work $(-11.5 \%)$ (Table 1$)$.

All variables analyzed in the cardiopulmonary exercise incremental test $\left(\dot{\mathrm{V}}_{2}, \dot{\mathrm{V}}_{\mathrm{E}}, \dot{\mathrm{V}}_{\mathrm{E}} / \dot{\mathrm{VO}}_{2}, \mathrm{Ti}\right.$, Te, $\mathrm{V}_{\mathrm{T}}, \mathrm{V}_{\mathrm{T}} / \mathrm{Ti}, f, \mathrm{HR}$, and workload) were similar for both limbs at each of the different exercise intensities (moderate, anaerobic threshold workload, and peak effort) (Table 2). 
Table 1. Muscle characteristics obtained from involved and uninvolved limbs four months after anterior cruciate ligament reconstruction surgery.

\begin{tabular}{ccccccc}
\hline & \multicolumn{2}{c}{ Involved } & \multicolumn{2}{c}{ Uninvolved } & \multicolumn{2}{c}{ p value } \\
& Mean & SD & Mean & SD & & \\
Thigh circumference $(\mathrm{cm})$ & 46.9 & 4.3 & 48.5 & 3.9 & 0.005 & 0.38 \\
Range of motion (degrees) & 126.1 & 5.2 & 129.3 & 5.5 & 0.01 & 0.59 \\
Knee extensor peak torque (Nm) & 116.5 & 29.1 & 220.8 & 40.4 & 0.00000 & 2.9 \\
Knee extensor total work $(\mathrm{J})$ & 135.2 & 31.2 & 237.5 & 57.1 & 0.00001 & 2.22 \\
Knee flexor peak torque $(\mathrm{Nm})$ & 107.8 & 15.4 & 123.5 & 26.3 & 0.004 & 0.72 \\
Knee flexor total work $(\mathrm{J})$ & 129.5 & 24.7 & 146.3 & 36.7 & 0.01 & 0.53 \\
\hline
\end{tabular}

Table 2. Cardiorespiratory responses to unilateral exercise test for the involved and uninvolved limbs during moderate, anaerobic threshold (AnT), and peak effort.

\begin{tabular}{|c|c|c|c|c|c|c|c|c|c|c|c|c|c|c|c|c|c|c|}
\hline \multirow[b]{2}{*}{ Moderate } & \multicolumn{2}{|c|}{$\begin{array}{c}\text { HR } \\
\text { beats.min }\end{array}$} & \multicolumn{2}{|c|}{$\begin{array}{c}\dot{\mathbf{V O}}_{2} \\
\text { L.min }\end{array}$} & \multicolumn{2}{|c|}{$\begin{array}{c}\text { VE } \\
\text { L.min }\end{array}$} & \multicolumn{2}{|c|}{$\dot{\mathbf{V E}} / \dot{\mathrm{V}}_{2}$} & \multicolumn{2}{|c|}{$\underset{\mathbf{b p m}}{f}$} & \multicolumn{2}{|c|}{$\begin{array}{l}\mathbf{V}_{\mathrm{T}} \\
\mathbf{L}\end{array}$} & \multicolumn{2}{|c|}{$\begin{array}{c}\mathbf{T i} \\
\mathbf{S}\end{array}$} & \multicolumn{2}{|c|}{$\begin{array}{c}\text { Te } \\
\mathrm{S}\end{array}$} & \multicolumn{2}{|c|}{$\begin{array}{c}\mathbf{V}_{\mathrm{T}} / \mathbf{T i} \\
\mathbf{L}^{-\mathrm{s}^{-1}}\end{array}$} \\
\hline & Mean & SD & Mean & SD & Mean & SD & Mean & SD & Mean & SD & Mean & SD & Mean & SD & Mean & SD & Mean & SD \\
\hline Uninvolved & 90 & 14 & 0.58 & 0.25 & 18.9 & 5.4 & 37 & 14 & 27 & 5 & 0.7 & 0.1 & 1.1 & 0.3 & 1.2 & 0.3 & 0.7 & 0.2 \\
\hline Involved & 88 & 12 & 0.57 & 0.20 & 18.4 & 5.1 & 36 & 13 & 29 & 7 & 0.7 & 0.2 & 1.1 & 0.3 & 1.2 & 0.3 & 0.7 & 0.2 \\
\hline \multicolumn{19}{|l|}{ AnT } \\
\hline Uninvolved & 105 & 16 & 0.98 & 0.34 & 26.4 & 5.5 & 27 & 6 & 27 & 6 & 1.0 & 0.3 & 1.1 & 0.3 & 1.2 & 0.3 & 0.9 & 0.2 \\
\hline Involved & 104 & 14 & 0.96 & 0.22 & 25.6 & 5.1 & 27 & 6 & 29 & 4 & 1.0 & 0.3 & 1.2 & 0.5 & 1.1 & 0.2 & 0.9 & 0.2 \\
\hline \multicolumn{19}{|l|}{ Peak } \\
\hline Uninvolved & 141 & 21 & 1.75 & 0.45 & 63.6 & 19.6 & 37 & 8 & 41 & 11 & 1.6 & 0.4 & 0.9 & 0.3 & 0.7 & 0.2 & 2.0 & 0.7 \\
\hline Involved & 137 & 21 & 1.81 & 0.47 & 62.1 & 17.9 & 35 & 8 & 41 & 10 & 1.6 & 0.6 & 0.8 & 0.2 & 0.7 & 0.2 & 1.9 & 0.5 \\
\hline
\end{tabular}

$\mathrm{HR}$ : heart rate; $\mathrm{VO}_{2}$ : oxygen consumption; VE: minute ventilation; $f$ : breath rate; $\mathrm{V}_{\mathrm{T}}$ : tidal volume; Ti: inspiratory time; Te: expiratory time; $\mathrm{V}_{\mathrm{T}} / \mathrm{Ti}$ : tidal volume/inspiratory time.

\section{Discussion}

This is the first study to examine the effects of ACL reconstruction surgery on cardiorespiratory responses to isometabolic exercise performed unilaterally. We found that, despite prominent impairment to muscle function (i.e. decrease in isokinetic muscle strength in involved limb compared with uninvolved limb), the cardiorespiratory responses to exercise were similar. This is a novel result because previous studies that investigated the effects of different muscle masses on physiological responses to exercise (one vs. two leg exercise ${ }^{15}$, upper vs. lower limb exercise ${ }^{16}$, and systemic illnesses that affect muscle mass such as chronic heart failure ${ }^{10}$ ) found that smaller muscle masses present more exacerbated physiological responses to isometabolic exercise.

In the present study on muscle function four months after ACL reconstruction, the involved limb presented a smaller thigh circumference than the uninvolved limb due to the muscle atrophy process after ligament reconstruction that resulted in a decrease in muscle work capacity and contraction, thus leading to lower values for knee extensor and flexor isokinetic torque.

In fact, quadriceps hypotrophy is a phenomenon expected in patients in the postoperative phase of ACL reconstruction, and higher deficit levels were expected after ACL reconstruction with patellar tendon than ACL reconstruction with hamstring graft, even though no difference was found between the two surgical procedures (patellar tendon graft or hamstring graft) over two years after surgery ${ }^{20}$. Concerning studies including quadriceps strength measurements, some authors showed that the contralateral deficit between limbs can still be seen up to two years after surgery ${ }^{21}$. Nevertheless, this strength deficit seems to have no correlation with other variables such as functional level and residual ligament laxity, indicating a lack of evidence of direct dependence on muscle response ${ }^{21}$. Similarly, our study did not find dependence between muscle impairment due to ACL reconstruction surgery and physiological responses to exercise. 
Long periods of skeletal muscle inactivity (due to limb immobilization, bed rest, physical inactivity or space flight) result in a myriad of physiological adaptations in skeletal muscle form and function that clinically manifest themselves as losses in muscle performance and require time-consuming physical rehabilitation ${ }^{22}$. In this context, immobilization is a model widely used to study skeletal muscle wasting that causes similar muscle repercussion to that of ACL reconstruction.

Skeletal muscle hypotrophy can occur due to a decrease in protein synthesis, an increase in the rate of protein degradation or a combination of both increased proteolysis and depressed protein synthesis. In animal models of disuse atrophy, it has been shown that inactivity-induced muscle hypotrophy occurs due to a decrease in protein synthesis and an increase in the rate of proteolysis ${ }^{23}$. In the hindlimb suspension model of skeletal muscle atrophy, the rate of protein synthesis declines rapidly following the onset of muscle unloading. This decrease in muscle protein synthesis reaches a new steady-state level at roughly 48 hours $^{23}$. Further, the reduction in protein synthesis is followed by a large and rapid increase in proteolysis mediated by several key proteases.

Moreover, skeletal muscle atrophy and weakness can occur due to neurological and skeletal muscle impaired properties, as it is well known that the output from these sources controls voluntary force production $^{24}$. Concerning the nervous system for instance, Seki et al. ${ }^{25}$ reported that the mean firing rate of the motor neuron in the first dorsal interosseous during maximal voluntary contraction decreased $15 \%$ after 1 week of finger immobilization. It is likely that the relative contribution of neural and muscular factors dynamically changes over time, with the muscular component contributing more as the duration of disuse increases ${ }^{26}$. Therefore, the muscle wasting after ACL reconstruction surgery can be attributed to the abovementioned aspects.

Because skeletal muscle is a plastic tissue that responds to increased or decreased contractile activity after ACL reconstruction surgery, its spontaneous recovery process can be amplified by rehabilitation. In an animal model study, Desplanches et al. ${ }^{27}$ compared the effects of spontaneous recovery and treadmill retraining for 8 weeks on $\dot{\mathrm{V}}_{2}$ max, histochemical, and biochemical muscular properties. The rats were submitted to 5 weeks of hindlimb suspension. The authors found that spontaneous recovery reversed $15 \%$ of the decrease in $\mathrm{VO}_{2}$ max, whereas retraining induced a $20 \%$ rise above control values. In the spontaneous recovery group, both citrate synthase and 3-hydroxyacyl-CoA dehydrogenase activities, decreased by hypokinesia $(-40 \%)$, increased but remained $20 \%$ below the control level, respectively. In the post-hypokinesia training group, there was a rise in these activities above control values $(+50$ and $+20 \%$, respectively).

Recovery or training led to $100 \%$ distribution of type I fiber in the soleus muscle and to a full recovery of all fiber cross-sectional areas. In the spontaneous recovery group, the amount of capillaries per fiber decreased by $46 \%$, returning to the normal range. In the post-hypokinesia group, training induced a rise in the capillaries per fiber above their control values $(+23 \%)$. These results point to the plasticity of the muscle and indicate the need of a post-hypokinesia training program for the full recovery of oxidative enzyme capacity.

In another study, Witzmann et al. ${ }^{28}$ studied contractile properties in rats remobilized after 6 weeks of hindlimb immobilization to evaluate the regenerative capacity of fast and slow skeletal muscle. The authors found that slow skeletal muscle was more sensitive to immobilization. However, its recovery rate was greater, occurring earlier than the fast muscle recovery. According to the authors, this is probably the result of higher protein turnover rates in slow skeletal muscle.

Therefore, this can be a possible explanation for our results. As the cardiorespiratory responses to exercise evaluated by the present study are related to aerobic metabolism, it is possible that oxidative status recovery (i.e. oxidative metabolism enzymes recovery) had happened earlier. In addition, during the first months of the rehabilitation process after ACL surgery, the patient needs to avoid heavy weight exercises, because it may be harmful to the new ligament graft and it may create an inflammatory process in the weakened quadriceps tendon. This type of exercise, particularly eccentric contraction ${ }^{29}$, has been shown to increase strength recovery but it was not emphasized in the first months after surgery. Therefore, it is possible that the oxidative status recovery had occurred before the strength status recovery. Likewise, fast skeletal muscle did not recover completely, which would explain the differences related to muscle strength.

\section{Limitations}

In this study, we showed that there was no difference in cardiorespiratory responses to isometabolic exercise after a 4-month rehabilitation program post-ACL reconstruction because of the significant loss in strength in the involved lower 
limb. However, this study was designed to begin evaluations 4 months after surgery, and future studies with earlier evaluations could show significant cardiorespiratory differences that may be useful in the rehabilitation process. Another possible limitation of this study was the fact that the strength evaluation was done in an open kinetic chain and the muscles involved in the test were only the knee flexors and extensors. On the other hand, in the cardiopulmonary incremental exercise test all lower limb muscles were involved as it was done in a closed kinetic chain. It is possible that the strength deficit of the extensor muscles was minimized in the cardiopulmonary incremental exercise test by the other muscular actions.

\section{Clinical implications}

After 4 months of rehabilitation post-ACL reconstruction, there are no differences in cardiorespiratory adjustments to dynamic exercise performed by both uninvolved and involved lower limbs after unilateral ACL reconstruction. The limb muscles may not present differences in oxidative and peripheral perfusion capacities that could elicit higher levels of peripheral cardiorespiratory stimulus during exercise because of the significant weakness of the knee extensor muscles. These results can be useful to guide the rehabilitation program for this very common knee ligament injury in orthopedic and sports settings.

\section{- Acknowledgements}

The volunteers who agreed to participate in this study.

This research was supported by Fundação de Amparo à Pesquisa do Estado de São Paulo (FAPESP), São Paulo, SP, Brazil, through grant no. 99/07746-9.

\section{References}

1. Whipp BJ, Ward SA. Determinants and control of breathing during muscular exercise. Br J Sports Med. 1998;32(3):199-211. http://dx.doi.org/10.1136/ bjsm.32.3.199

2. Aminoff T. Prediction of acceptable physical work loads based on responses to prolonged arm and leg exercise. Ergomonics. 1998;41(1):109-20. PMid:9468809. http:// dx.doi.org/10.1080/001401398187350

3. Innes JA, De Cort SC, Evans PJ, Guz A. Central command influences cardiorespiratory response to dynamic exercise in humans with unilateral weakness. J Physiol. 1992;448:551-63. PMid:1593477.

4. Ward SA. Control of the exercise hyperpnoea in humans: a modeling perspective. Resp Physiol. 2000;122(2-3):14966. http://dx.doi.org/10.1016/S0034-5687(00)00156-0

5. Waldrop TG, Eldridge FL, Iwamoto GA, Mitchell JH. Central neural control of respiration and circulation during exercise. In: Rowell LB, Shepherd JT, editors. Handbook of Physiology. Section 17: Exercise: Regulation and Integration of Multiple Systems. New York: Oxford University Press; 1996. p. 333-80.

6. Gandevia SC, Macefield VG, Bigland-Ritchie B, Gorman $\mathrm{RB}$, Burke D. Motoneuronal output and gradation of effort in attempts to contract acutely paralysed leg muscles in man. J Physiol. 1993;471:411-27. PMid:8120814.

7. Wuyam B, Moosavi SH, Decety J, Adams L, Lansing RW, Guz A. Imagination of dynamic exercise produced ventilatory responses which were more apparent in competitive sportsmen. J Physiol. 1995;482(Pt 3):713-24. PMid:7738860.

8. Wasserman K, Hansen JE, Sue DY, Casaburi R, Whipp BJ. Principles of Exercise Testing and Interpretation. 3rd ed. Baltimore: Maryland Lippincott Williams \& Wilkins; 1999. p. 47-8.

9. Mateika JH, Duffin J. A review of the control of breathing during exercise. Eur J Appl Physiol Occup Physiol. 1995;71(1):1-27. PMID: 7556128. http://dx.doi. org/10.1007/BF00511228

10. Piepoli MF, Kaczmarek A, Francis DP, Davies LC, Rauchhaus M, Jankowska EA, et al. Reduced peripheral skeletal muscle mass and abnormal reflex physiology in chronic heart failure. Circulation. 2006;114(2):12634. PMid:16818813. http://dx.doi.org/10.1161/ CIRCULATIONAHA.105.605980

11. Huszczuk A, Yeh E, Innes JA, Solarte I, Wasserman K, Whipp BJ. Role of muscle perfusion and baroreception in the hyperpnea following muscle contraction in dog. Respir Physiol. 1993;91(2-3):207-26. http://dx.doi. org/10.1016/0034-5687(93)90100-O

12. Garrison JC, Shanley E, Thigpen C, Geary R, Osler $\mathrm{M}$, Delgiorno J. The reliability of the vail sport test as a measure of physical performance following anterior cruciate ligamentreconstruction. Int J Sports Phys Ther. 2012;7(1):20-30. PMid:22389868 PMCid:PMC3290923.

13. Andrade MS, Cohen M, Piçarro IC, Silva AC. Knee performance after anterior cruciate ligament reconstruction. Isokinetics and Exercise Science. 2002;10(2):81-6.

14. Thomas AC, Villwock M, Wojtys EM, Palmieri-Smith RM. Lower Extremity Muscle Strength After Anterior Cruciate Ligament Injury and Reconstruction. J Athl Train. 2013 [Epub ahead of print]. PMID: 23725517. http://dx.doi. org/10.4085/1062-6050-48.3.23

15. Magnusson G, Kaijser L, Isberg B, Saltin B. Cardiovascular responses during one- and two-legged exercise in middle-aged men. Acta Physiol Scand. 1994;150(4):353-62. PMid:8036904. http://dx.doi. org/10.1111/j.1748-1716.1994.tb09699.x

16. Ishida K, Takaishi T, Miyamura M. Ventilatory responses at the onset of passive movement and voluntary 
exercise with arms and legs. Acta Physiol Scand. 1994;151(3):343-52. PMid:7976406. http://dx.doi. org/10.1111/j.1748-1716.1994.tb09753.x

17. Carey T, Oliver D, Pniewski J, Mueller T, Bojescul J. Anterior cruciate ligament augmentation for rotational instability following primary reconstruction with an accelerated physical therapy protocol. J Surg Orthop Adv. 2013;22(1):59-65. PMid:23449057. http://dx.doi. org/10.3113/JSOA.2013.0059

18. Andrade MS, De Lira CA, Koffes FC, Mascarin NC, Benedito-Silva AA, Da Silva AC. Isokinetic hamstringsto-quadriceps peak torque ratio: the influence of sport modality, gender, and angular velocity. J Sports Sci. 2012;30(6):547-53. PMid:22364375. http://dx.doi.org/1 $0.1080 / 02640414.2011 .644249$

19. Gleeson NP, Mercer TH. Reproducibility of isokinetic leg strength and endurance characteristics of adult men and women. Eur J Appl Physiol Occup Physiol. 1992;65:221-8. http://dx.doi.org/10.1007/BF00705085

20. Dauty M, Tortellier L, Rochcongar P. Isokinetic and anterior cruciate ligament reconstruction with hamstrings or patella tendon graft: analysis of literature. Int J Sports Med. 2005;26(7):599-606. PMid:16195995. http://dx.doi. org/10.1055/s-2004-821342

21. Mattacola CG, Perrin DH, Gansneder BM, Gieck JH, Saliba EN, McCue FC 3rd. Strength, functional outcome, and postural stability after Strength, functional outcome, and postural stability after reconstruction. J Athl Train. 2002;37(3):262-8. PMid:12937583 PMCid:PMC164354.

22. Booth FW. Effect of limb immobilization on skeletal muscle. J Appl Physiol. 1982;52(5):1113-8. PMid:7047468.

23. Thomason DB, Biggs RB, Booth FW. Protein metabolism and beta-myosin heavy-chain mRNA in unweighted soleus muscle. Am J Physiol. 1989;257(2Pt2):R300-5. PMid:2764153.
24. Clark BC, Manini TM. Sarcopenia $\neq$ dynapenia. J Gerontol A Biol Sci Med Sci. 2008;63(8):829-34. http://dx.doi. org/10.1093/gerona/63.8.829

25. Seki K, Kizuka T, Yamada H. Reduction in maximal firing rate of motoneurons after 1-week immobilization of finger muscle in human subjects. J Electromyogr Kinesiol. 2007;17(2):113-20. PMid:16448820. http:// dx.doi.org/10.1016/j.jelekin.2005.10.008

26. De Boer MD, Maganaris CN, Seynnes OR, Rennie MJ, Narici MV. Time course of muscular, neural and tendinous adaptations to 23 day unilateral lower-limb suspension in young men. J Physiol. 2007;583(Pt3):107991. PMid:17656438 PMCid:PMC2277190. http://dx.doi. org/10.1113/jphysiol.2007.135392

27. Desplanches D, Mayet MH, Semporé B, Frutoso J, Flandrois R. Effect of spontaneous recovery or retraining after hindlimb suspension on aerobic capacity. J Appl Physiol. 1987;63(5):1739-43. PMid:3693209.

28. Witzmann FA, Kim DH, Fitts RH. Recovery time course in contractile function of fast and slow skeletal muscle after hindlimb immobilization. J Appl Physiol. 1982;52(3):67782. PMid:7068483.

29. Brasileiro JS, Pinto OM, Avila MA, Salvini TF. Functional and morphological changes in the quadriceps muscle induced by eccentric training after ACL reconstruction. Rev Bras Fisioter. 2011;15(4):284-90. PMid:21860991. http://dx.doi.org/10.1590/S1413-35552011005000012

\section{Correspondence}

\section{Marília dos Santos Andrade}

Universidade Federal de São Paulo (UNIFESP)

Departamento de Fisiologia

Rua Botucatu, 862, $5^{\circ}$ andar, Edifício de Ciências Biomédicas,

Vila Clementino

CEP 04023-900, São Paulo, SP, Brazil

e-mail: marilia.andrade@unifesp.br 